\title{
Analysis of fractal radiation patterns from concentric-ring hexagonal arrays
}

\author{
A. Boualleg, N. Merabtine, M. Benslama \\ Laboratory of Electromagnetism and Telecommunications LET, Department of Electronics, \\ University Mentouri Constantine, Algeria \\ E-mail:bouadzdz@yahoo.fr; na_merabtine@hotmail.com; malekbenslama@hotmail.com
}

\begin{abstract}
These hexagonal arrays are becoming increasingly popular, especially for their applications in the area of wireless communications. The overall objective of this article has been to use the theoretical foundation developed for the analysis of radiation patterns and design of the hexagonal arrays. A technique has been developed for the analysis of radiation patterns from concentric ring arrays. A family of functions, known as generalized Weierstrass functions, has been shown to play a key role in the theory of fractal radiation pattern analysis.
\end{abstract}

Keywords: fractal antenna arrays, fractal antenna radiation patterns, low side-lobe antennas radiation patterns.

Manuscript received 30.09.05; accepted for publication 25.10.05.

\section{Introduction}

The name "fractal", from the Latin "fractus" meaning broken, was given to highly irregular sets by Benoit Mandelbrot in his foundational essay in 1975 [1]. Since then, fractal geometry has attracted widespread, and sometimes controversial, attention. The subject has grown on two fronts: on the one hand, many "real fractals" of science and nature have been identified. On the other hand, mathematics that is available for studying fractal sets, a lot of which has its roots in geometric measure theory, has developed enormously with new tools emerging for fractal analysis. This paper concerned with mathematics of fractals and application to the antenna theory [2].

\section{Theory}

The standard hexagonal arrays are formed by placing elements in equilateral triangular grid with spacings $d$. These arrays can also be rounded by several concentric six-element circular arrays of different radii [3, 4]. The resulting expression for the hexagonal array factor, in a normalized form, is given by [2].

$$
F_{P}(\theta, \varphi)=\frac{I_{0}+\sum_{p=1}^{P} \sum_{m=1}^{P} \sum_{n=0}^{5} I_{p m n} e^{j\left[k r_{p m} \sin \theta \cos \left(\varphi-\varphi_{p m n}\right)+\alpha_{p m n}\right]}}{I_{0}+\sum_{p=1}^{P} \sum_{m=1}^{P} \sum_{n=0}^{5} I_{p m n}},
$$

where

$$
\begin{aligned}
& r_{p m}=d \sqrt{p^{2}+(m-1)^{2}-p(m-1)}, \\
& \varphi_{p m n}=\cos ^{-1}\left[\frac{r_{p m}^{2}+d^{2} p^{2}-d^{2}(m-1)^{2}}{2 r_{p m} d p}\right]+\frac{n p}{3}, \\
& \alpha_{p m n}=-k r_{p m} \sin \theta_{0} \cos \left(\varphi_{0}-\varphi_{p m n}\right)
\end{aligned}
$$

and $P$ is the number of concentric hexagons in the array. Hence, the total number of elements contained in an array with $P$ hexagons is

$N_{P}=3 P(P+1)+1$.

At this point: we investigate the possibility that useful designs for hexagonal arrays may be realized via a construction process based on the recursive application of a generating subarray. To demonstrate this, suppose we consider the uniformly excited six-element circular generating subarray of the radius $r=\lambda / 2$, shown in Fig. 1.

This particular value of the radius was chosen so that these six elements in the array correspond to the vertices of a hexagon with half-wavelength sides (i. e., $r=\lambda / 2$ ). Consequently, the array factor associated with this sixelement generating subarray may be shown to have the following representation:

$$
F_{P}(\theta, \varphi)=\frac{1}{6^{P}} \prod_{p=1}^{P} \sum_{n=1}^{6} e^{j \delta^{P-1}\left[\pi \sin \theta \cos \left(\varphi-\varphi_{n}\right)+\alpha_{n}\right]},
$$




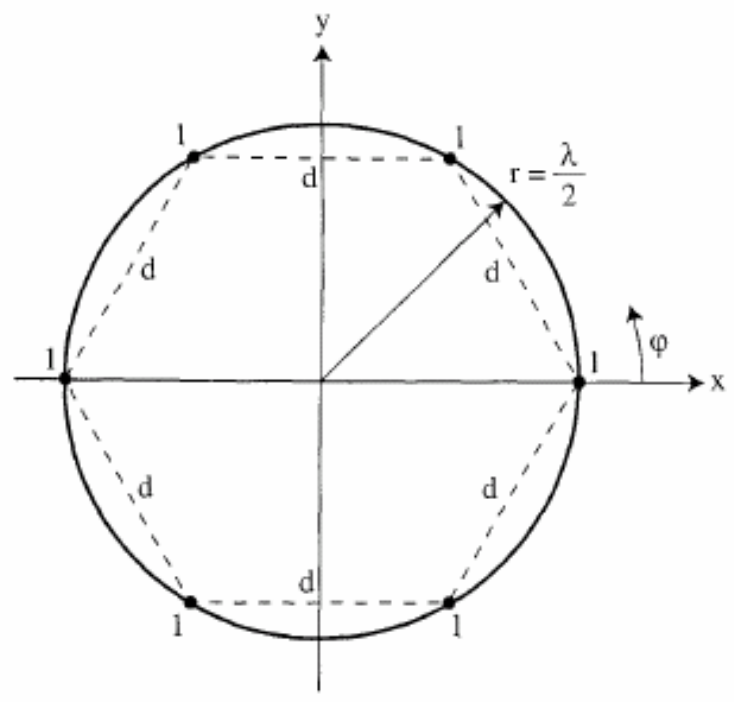

Fig. 1. The geometry for a uniformly excited six-element circular subarray generator of the radius $r=\lambda / 2$.

where

$\varphi_{n}=(n-1) \frac{\pi}{3}, \alpha_{n}=-\pi \sin \theta_{0} \cos \left(\varphi_{0}-\varphi_{n}\right)$.

The array factor expression given in (2) may also be written in the form

$$
F_{P}(\theta, \varphi)=\frac{1}{6^{P}} \prod_{p=1}^{P} \sum_{n=1}^{6} e^{j \delta^{P-1} \psi_{n}(\theta, \varphi)},
$$

where

$$
\psi_{n}(\theta, \varphi)=\pi\left[\sin \theta \cos \left(\varphi-\varphi_{n}\right)-\sin \theta_{0} \cos \left(\varphi_{0}-\varphi_{n}\right)\right] .
$$

Stage 1: $N_{P}=6$

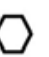

Stage 2: $N_{P}=30$ The number of elements thinned $=240$

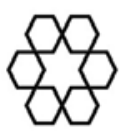

Stage 3: $N_{P}=132$ The number of elements thinned $=240$
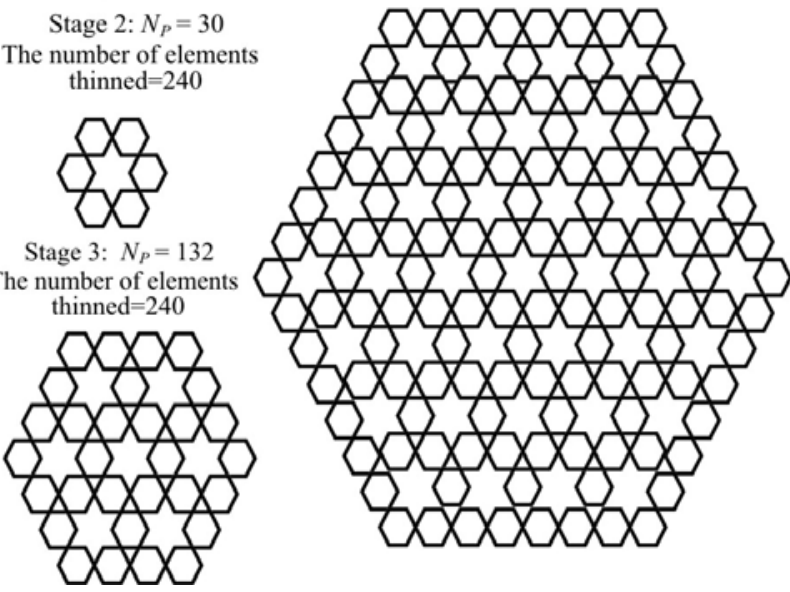

Fig. 2. The first four stages in the construction of a hexagonal array via the generating subarray illustrated in Fig. 1, with the expansion factor $\delta=2$.The element locations correspond to the vertices of the hexagons.
We will first examine the special case where the expansion factor of the recursive hexagonal array is assumed to be unity, i.e., $\delta=1$. Under these circumstances, equation (3) reduces to

$F_{P}(\theta, \varphi)=\left[\frac{1}{6} \sum_{n=1}^{6} e^{j \psi_{n}(\theta, \varphi)}\right]^{P}$.

These arrays increase in size at a rate that obeys the relationship

$N_{P}=3 P(P+1)+\left(1-\delta_{p l}\right)$,

where $\delta_{p l}$ represents the Kronecker delta function defined by [2]

$\delta_{p l}=\left\{\begin{array}{ll}1, & P=1 \\ 0 & P \neq 1\end{array}\right.$.

In other words, every time this fractal array evolves from one stage to the next, the number of concentric hexagonal subarray contained in it increases by one.

The second special case of interest to be considered in this section results when a choice of $\delta=2$ is made. Substituting this value of $\delta$ into (3) yields an expression for the recursive hexagonal array factor given by

$F_{P}(\theta, \varphi)=\frac{1}{6^{P}} \prod_{p=1}^{P} \sum_{n=1}^{6} e^{j 2^{P-1} \psi_{n}(\theta, \varphi)}$,

where

$N_{P}=3\left[2^{P}\left(2^{P}-1\right)-2^{P-1}\left(2^{P-1}-1\right)\right]$.

Clearly, by comparing $N_{P}$ (for $\delta=2$ ) with $N_{P}$ (for $\delta=1$ ), we conclude that these recursive arrays will grow at a much faster rate than those generated by a choice of $\delta=1$. The representations of the first four stages in the construction process of these arrays are illustrated in Fig. 2, where the element locations correspond to the vertices of the hexagons.

Fig. 2 indicates that the hexagonal arrays resulting from the recursive construction process with $\delta=2$ have some elements missing, i. e., they are thinned.

It is interesting to look what happens with these arrays when an element with two units of current is added to the center of the hexagonal generating subarray shown in Fig. 1. Under these circumstances, the expression for the array factor given in (3) must be modified in the following way:

$F_{P}(\theta, \varphi)=\frac{1}{8^{P}} \prod_{p=1}^{P}\left\{2+\sum_{n=1}^{6} e^{j \delta^{p-1} \psi_{n}(\theta, \varphi)}\right\}$.

Plots of a several radiation patterns calculated from (6) with $\delta=1$ and $\delta=2$ are shown in Figs 3 and 4, respectively. It is evident from Fig. 4 that the radiation patterns for these arrays have no side lobes. 
Semiconductor Physics, Quantum Electronics \& Optoelectronics, 2005. V. 8, N 4. P. 91-94.
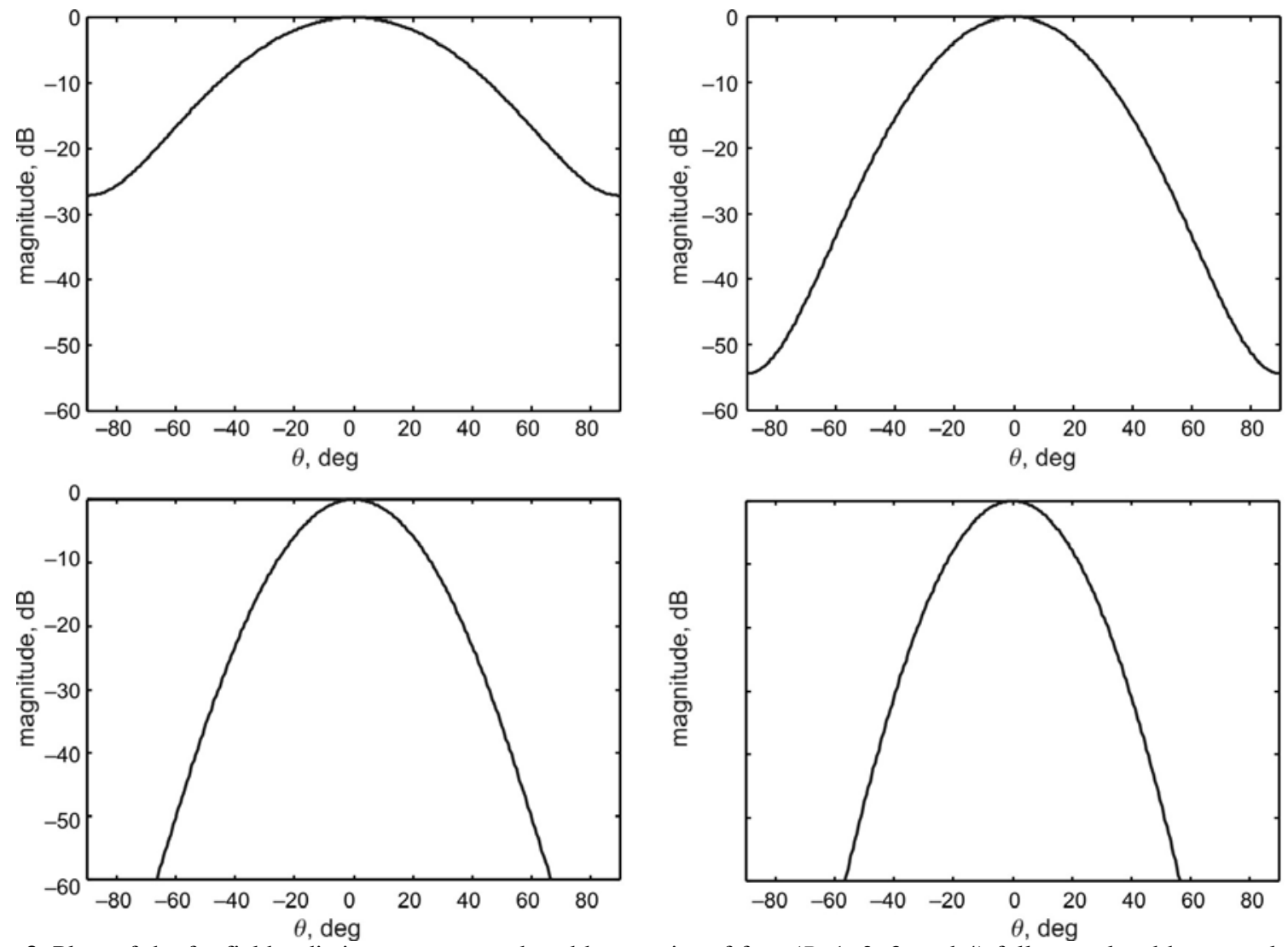

Fig. 3. Plots of the far field radiation patterns produced by a series of four $(P=1,2,3$, and 4$)$ fully populated hexagonal arrays generated with the expansion factor $\delta=1$.
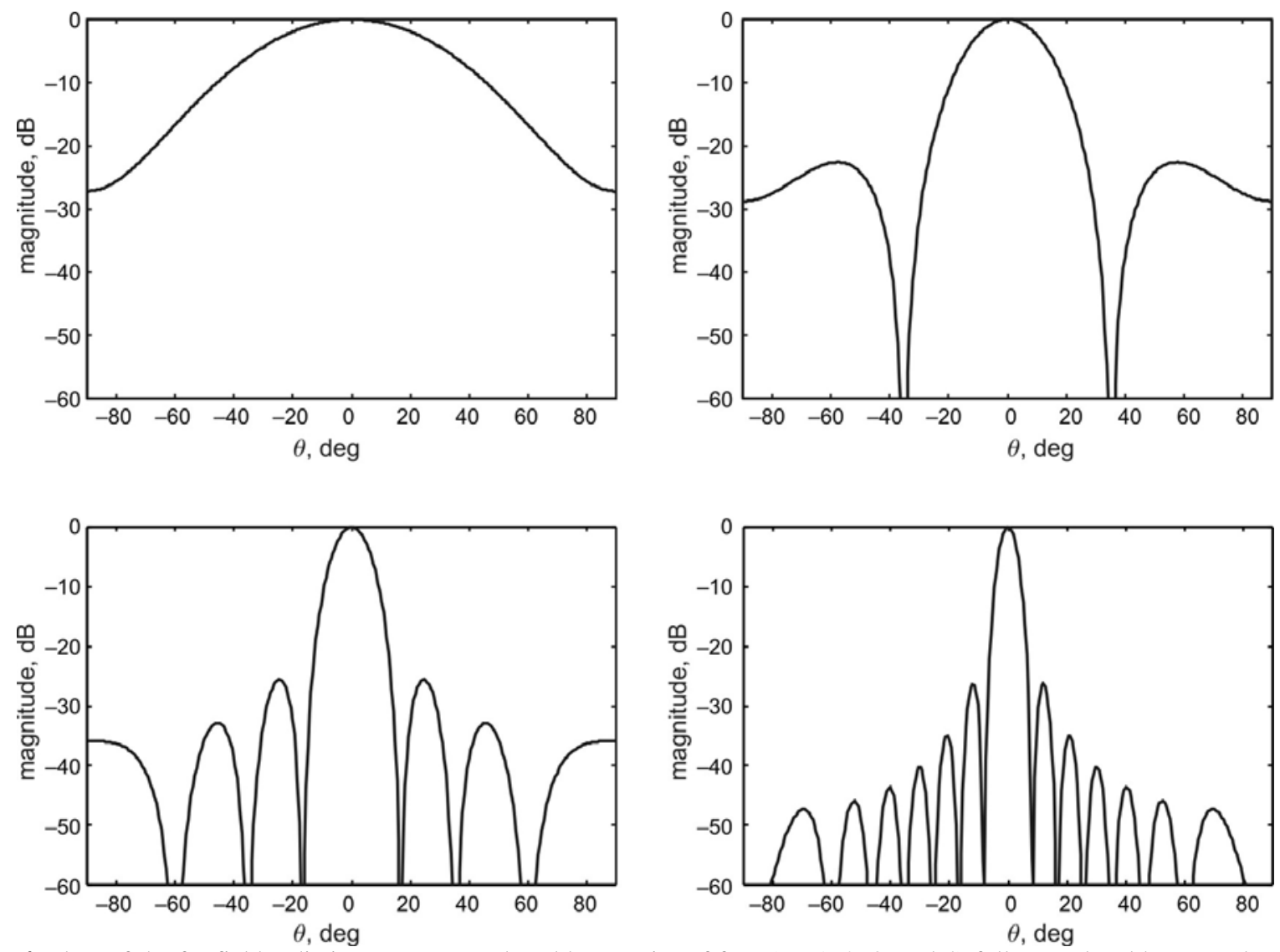

Fig. 4. Plots of the far field radiation patterns produced by a series of four $(P=1,2,3$, and 4) fully populated hexagonal arrays generated with the expansion factor $\delta=2$.

(C) 2005, V. Lashkaryov Institute of Semiconductor Physics, National Academy of Sciences of Ukraine 
These plots indicate that a further reduction in side-lobe levels may be achieved by including a central element in the generating subarray of Fig. 1.

\section{Conclusion}

The research in the area of fractal antenna has recently yielded a rich class of new designs for antenna elements as well as arrays.

The essential property of hexagonal arrays have some elements missing, i. e., they are thinning. This is a potential advantage of these arrays from the design point of view, since they may be realized with fewer elements. Another property of these arrays is that they possess low side-lobe levels at $\varphi=\pi / 2$. Finally, it should be noted that the compact product form of the array factor can be obtained for some particular cases. This offers a significant advantage in terms of computational efficiency, especially for large arrays, and may be exploited to develop rapid beam-forming algorithms.

\section{References}

1. B.B. Mandelbrot, The fractal geometry of nature. San Francisco (1975).

2. D.H. Werner, R.L. Haupt and P.L. Werner, Fractal antenna engineering: The theory and design of fractal Antenna Arrays // IEEE Antennas and Propagation Magazine 41, No 5, p. 37-59 (1999).

3. V.F. Kravchenko, and V.M. Masyuk, Analysis and synthesis of multiband antenna arrays // Electromagnetic waves and electronic systems 9, Nos 3-4 (2004).

4. V.F. Kravchenko, and V.M. Masyuk, The ring fractal antenna arrays // Electromagnetic waves and electronic systems 9, No 5 (2004). 